\title{
Psychological Experiments and Phenomenal Experience in Size and Shape Constancy
}

\author{
Gary Hatfield*i
}

Some experiments in perceptual psychology measure perceivers' phenomenal experiences of objects versus their cognitive assessments of object properties. Analyzing such experiments, this article responds to Pizlo's claim that much work on shape constancy before 1985 confused problems of shape ambiguity with problems of shape constancy. Pizlo fails to grasp the logic of experimental designs directed toward phenomenal aspects of shape constancy. In the domain of size perception, Granrud's studies of size constancy in children and adults distinguish phenomenal from cognitive factors.

1. Introduction. This paper concerns the interaction between theoretical aims in the study of perception and the experimental design of a type of perceptual experiment. It focuses on experiments that aim to measure perceivers' phenomenal experiences of objects in distinction from their cognitive assessments of object properties. It uses this distinction to respond to Pizlo's (2008) claim that nearly all work on the psychology of shape constancy prior to 1985 mistakenly confused problems of shape ambiguity with problems of shape constancy. I suggest instead that Pizlo has misunderstood the aim of work on phenomenal aspects of shape constancy and so has not grasped the relevant logic of experimental design. He assumes a different paradigm of shape perception - representing shape independent of viewpoint - that does not address how specific phenomenal experiences of shape arise and are related to objective shapes.

\footnotetext{
* To contact the author, please write to: Department of Philosophy, Cohen Hall 433, University of Pennsylvania, Philadelphia, PA 19104-6304; e-mail: hatfield@sas.upenn.edu.

$\dagger$ Thanks to Uljana Feest, Mazviita Chirimuuta, and Eric Schwitzgebel for comments at PSA12 and the members of the Seminar on Experimental Psychology and Phenomenology, École Normale Supérieure, Paris, for discussion of a subsequent version.
}

Philosophy of Science, 81 (December 2014) pp. 940-953. 0031-8248/2014/8105-0019\$10.00

Copyright 2014 by the Philosophy of Science Association. All rights reserved. 
Questions about the relation between phenomenal experience and cognitive factors also arise for size perception. Granrud's $(2004,2012)$ experimental investigations of size constancy in children and adults suggest that differences between younger children as opposed to older children and adults arise from cognitive development, not from a change in the experience of phenomenal size. In addition, I describe some everyday observations that support distinguishing phenomenal experiences of size from judgments about objective size.

In teasing apart phenomenal experience and objective judgments, investigators need not suppose that subjects ever experience a purely phenomenal perceptual state devoid of judgmental content. Nor need they assume that cognitive factors are absent from the processes that produce phenomenal experience. They need only suppose that phenomenal aspects of perceptual experience can be distinguished from beliefs about perceptual experience in some way or other. Experimental protocols are intended to permit such a distinction.

2. Aims and Methods of Experimentation in Perceptual Psychology. Perceptual theorists regularly distinguish between sensory and cognitive aspects of perception: between determinately phenomenal aspects of perceptual experience, such as shaped and colored surfaces, and recognition of those surfaces as bounding an object of a certain kind, say, one's favorite coffee mug. They thus distinguish between something's appearing circular and the recognition of the thing as a circle (a conceptual act of classification), or between its appearing red and its classification as a red thing. This distinction is exemplified in work on the perceptual constancies.

Empirical work on the perceptual constancies was a mainstay of twentiethcentury experimental psychology. "Perceptual constancy" refers to the fact that the phenomenal experience of various dimensions of perceptual stimulation is stable and constant in comparison with variation in the proximal values of those stimulus dimensions. In the words of perceptual psychologist Irvin Rock, "perceived qualities such as color, size, and the like tend to remain constant despite the fact that the proximal stimulus, for example, the retinal image of objects, is continually changing" $(1975,10)$. Focusing on vision, the constancies encompass the perception of color, lightness, size, and shape (among others). Objects appear to have a comparatively stable color under variations in illumination. Similarly, objects in space possessing a specific shape project a variety of shapes onto the retinas, depending on their orientation relative to the line of sight. Subjects report a comparatively stable shape across orientations. Even if the projected shapes are very similar (as in a family of ellipses presented at orientations that would yield equivalent perspective images), under many viewing conditions subjects report values closer to the objective shape of the distal stimulus than to the projective shape 
imaged on their retinas. Finally, objects do not appear to shrink as they move from near to farther away from an observer. An object at twice the distance does not appear half as tall, even though it takes up only half as large a visual angle. Phenomenally, objects typically do look somewhat smaller in the distance, yet we immediately recognize (cognitively) that they have not shrunk in size.

In studying the visual constancies, investigators have distinguished different aspects of subjects' responses to stimuli. Let us focus on shape constancy. Experimenters distinguish (1) constancy in the subjects' phenomenal experience of the distal stimulus and (2) constancy in subjects' perceptually based beliefs about the objective shape of that stimulus. (Experimenters are interested in other aspects of shape perception as well, but we may simplify by focusing on these two.) Let us refer to subjects' experience of the appearance of the shape, or their being presented with an "apparent shape," as aspect 1 and subjects' cognitive evaluation of "objective shape" as aspect 2. Experimenters manipulate various independent variables so as to determine their effects on one or the other or both of these aspects of shape perception. The independent variables include the shapes of the stimuli, their orientations to the line of sight, and the viewing conditions, including illumination, monocular or binocular viewing, the amount of viewing time allowed, whether the end of the stimulus presentation is followed by a dark field or a visual "mask," and so on. The dependent variable is the subjects' perceptual responses, as measured in one or another way.

Aspects 1 and 2 constitute different dependent variables. The first seeks to determine the subjects' experience of "apparent shape," whereas the second concerns a cognitive evaluation of "objective shape." A measurement procedure is used to assess these variables. Experimenters have devised several different ways of assessing the shape that a subject perceives on an experimental trial. A common method is to have the subject match the distal stimulus to a nearby comparison stimulus. The nearby stimulus might be allowed to vary in shape continuously, but more often the subject considers a series of nearby shapes and indicates which most closely matches the distal stimulus. If the distal stimulus were an ellipse, the subject would be allowed to choose from a page of elliptical shapes that were ordered in some way (e.g., the experimenter might randomly intersperse, from trial to trial, comparison stimuli ordered top to bottom on a page by ascending or descending values of eccentricity). The perceived value is then recorded as the value indicated by the subject; such values can be averaged over many trials for each experimental condition.

The comparison stimuli by themselves cannot distinguish aspects 1 and 2 above. In measuring those two aspects, the physical measuring device might be the same (a set of ellipses ordered on a page). How, then, can the experimenter determine values for aspects 1 and 2? In this case, the cog- 
nitive set of the subject becomes another independent variable, one that determines the type of measurement that occurs when the subject interacts with the comparison stimuli. The cognitive set is manipulated by giving the subjects different instructions at the outset of a block of trials. Subjects may be instructed to focus on the apparent shape, independently of their belief about the actual physical shape, or they may be instructed to indicate the objective shape that they believe the object to have on the basis of current perception. The first are called "apparent instructions," the second "objective instructions." (There are other instruction types, including "projective" instructions, under which the subject indicates the shape a stimulus would project onto a frontoparallel plane.)

How can experimenters be confident that the instructions are having the desired effect on the subject and thus on measurements of the subjects' objective or apparent perception? Several factors can come into play. Experiments are generally devised to test theories, and the theories provide a context for predicting the effects on subjects' responses of various manipulations of independent variables. To the extent that experimental data show the predicted pattern of responses under the two sets of instructions, these predictions are confirmed and the effectiveness of the measuring procedure is supported. One might also seek to calibrate the measures by having subjects respond to one of the two instructions under maximally good viewing conditions. In this case, objective and apparent instructions might be predicted to approach or coincide with the physical value of the stimulus. If they do, or if the deviations are systematic between the two conditions, then this initial calibration supports the effectiveness of the measuring procedure. Finally, in setting up the conditions, experimenters may consult their own experience of perceived objective and apparent shape; if subjects give responses that make sense in light of the experimenters' experiences, confidence in the measurements is bolstered.

In an investigation of phenomenal shape constancy, experimenters are faced with a challenge. They need to ensure that subjects are relying on their current perceptual experience in indicating what shape they perceive, rather than tacitly or unwittingly guessing the objective shape using background assumptions or indirect hints. In meeting this challenge, the experimenters must find a shape or family of shapes that, when presented to subjects, forces them to rely on their perceptual experience for a response, rather than guessing or inferring that one of a familiar set of shapes has been used. For this reason, many experiments on shape perception use a family of ellipses. These ellipses can be chosen so as to be equivalent to one another in their projective shapes when appropriately slanted relative to the line of sight. If experimenters chose squares and rectangles, these might be projectively equivalent with trapezoids, but subjects might well assume that a square or a rectangle is present and use that assumption to derive its unique slant and 
shape. There is no corresponding possibility for an ellipse, since its objective shape can be determined only by accurately perceiving its specific eccentricity. Unlike right quadrilaterals, ellipses do not come in a few standard shape types; they can vary continuously.

Experiments using ellipses show that, under good conditions of observation, such as binocular viewing of a stimulus illuminated for one-tenth of a second followed by darkness, subjects approach phenomenal constancy. Figure 1 summarizes the results of one such experiment. The investigators used two sets of projectively equivalent ellipses to ensure that subjects did not rely on knowledge of the available physical shapes, gained from earlier trials, to guess the shape cognitively. The aim was to have subjects rely on their just-ended phenomenal experience in providing a shape response. When the stimulus was succeeded by a visual mask, curtailing observation and processing time, constancy was, as expected, degraded toward projective values, and slant responses moved toward frontal values.

3. Pizlo's Challenge. Recently, Pizlo (2008) has challenged the design of nearly all previous empirical studies of shape constancy. He maintains that ellipses are not good stimuli because they vary on only one parameter (eccentricity); by contrast, right quadrilaterals have more internal structural constraints. If one assumes that a figure is a rectangle, then its projective shape directly yields information about its objective shape; the shape of a given rectangle's projected trapezoid directly specifies the degree of slant and relative length and width of the rectangle (again, assuming that the object is a rectangle). The choice of ellipses excludes such information (based on assumptions by the subject) for determining the objective shape.

Pizlo (2008) reviews the shape constancy literature stemming from Thouless $(1931,1932)$. Thouless investigated shape constancy for circles and squares. He rotated the circle about its diameter, and he rotated the square around a diagonal axis (corner to corner; rotation yielded a diamond). In a first experiment, subjects made drawings of the resulting shapes and so were not restricted to a circle or a square (they might draw ellipses or diamonds). In a subsequent experiment using only circles, subjects matched what they saw to a range of cardboard ellipses. In the 1950s and after, additional investigations were carried out by Leibowitz and Bourne (1956) using ellipses; by Epstein, Bontrager, and Park (1962) using triangles; and by others (see Pizlo 2008, 20) using stimuli that did not allow subjects to reduce uncertainty by guessing that it was an instance of a particular type of shape.

Pizlo $(2008,19-22)$ claims that nearly all this literature is flawed because of the use of ellipses (including circles) or triangles as stimuli. As we have seen, a given ellipse on the retina is projectively compatible with an infinity of ellipses in the world at varying slants. A slanted circle projects an ellipse, but a differently slanted ellipse can produce the same ellipse (fig. 2a). (A 

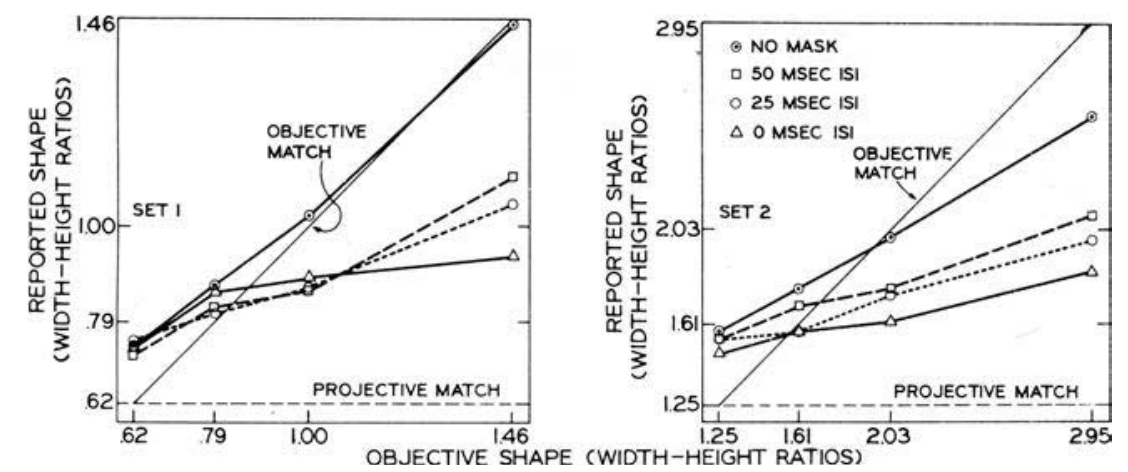

Figure 1. Perceived shape as a function of viewing condition (no mask for ca. $0.15 \mathrm{~s}$; masked for various interstimulus intervals or ISIs) for two sets of projectively equivalent ellipses under apparent viewing instructions. As the interval between mask and stimulus decreased, constancy degraded, as expected. Slant judgments also degraded with shorter intervals, as expected (but were less accurate). From Epstein, Hatfield, and Muise (1977), (C) American Psychological Association, reprinted with permission.

similar point holds for triangles.) By contrast, for a given quadrilateral on the retina, only one square or rectangle could produce it (leaving aside size). An infinity of quadrilaterals of varying shape might produce a given quadrilateral, but only one square or rectangle (fig. $2 b$ ). Hence, Pizlo suggests that, in a proper investigation of shape constancy, subjects should know that the shape in question is a square or rectangle (as in Stavrianos 1945), their only job being to determine which square or rectangle it is (Pizlo 2008, 24).

In Pizlo's reasoning, experiments using projectively equivalent ellipses are investigating shape ambiguity, not shape constancy. Accordingly, "ellipses must not be used to study shape constancy" (Pizlo 2008, 19). In section 2 I asserted that investigators advisedly chose projectively equivalent families of ellipses in order to ensure that they were investigating phenomenal shape constancy as opposed to a recognition task. So what has gone wrong?

The problem lies with Pizlo's understanding of shape constancy and of the aims of experimental work on biological subjects. Pizlo's own interest is in machine vision and machine recognition of objective shape. This led him to ignore phenomenal constancy.

Here is Pizlo's definition of shape constancy: "Note that when you view your car from a new angle, its image on your retina changes, but it is perceived as the same car. This fact defines what is called 'shape constancy.' Formally, 'shape constancy' refers to the fact that the percept of the shape of a given object remains constant despite changes in the shape of the object's retinal image" $(2008,3)$. The example of identifying your car from a new 
(a)

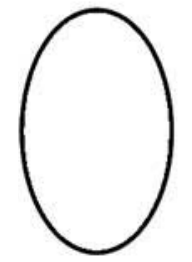

rotated: $0^{\circ}$

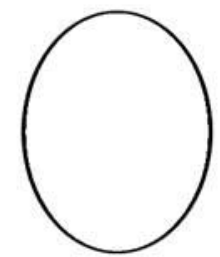

rotate: $39^{\circ}$

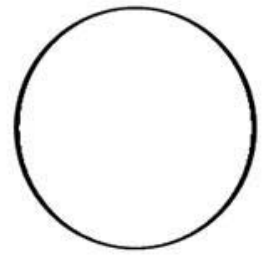

rotate: $52^{\circ}$

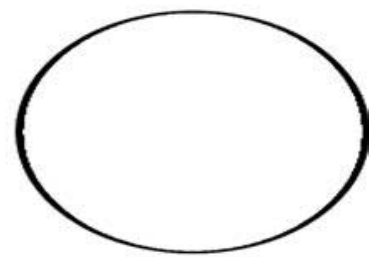

rotate: $65^{\circ}$

(b)
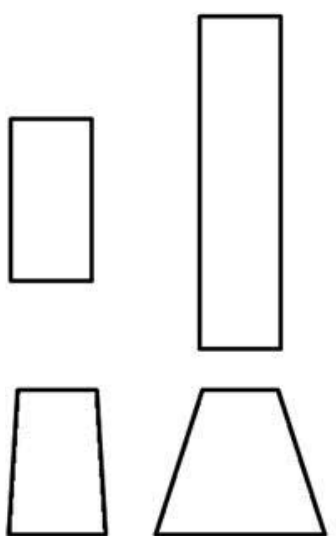

Figure 2. Shapes in $(a)$ are a set of ellipses that are projectively equivalent when the longer ones are increasingly rotated (by a specified amount). Shapes in $(b)$, if assumed to be rectangles, illustrate the fact that a unique shape is associated with the slant of a rectangle away from the frontal plane. If the figure is not known to be a rectangle, then the projective shape is consistent with a variety of trapezoids.

angle suggests that Pizlo is talking about the cognitive task of object identification- "same object again"-rather than the perceptual task of representing the shape of the car. However, the "formal" definition seems more promising, because it introduces the notion of the "percept" remaining the same across variations in the retinal image. So what is a "percept" for Pizlo?

The question is not easy to answer. Pizlo (2008) uses the term many times, but he never introduces it, and it does not appear in the book's index. We can get some sense of its meaning from examples.

In his usage, Pizlo easily slides from shape percept to shape recognition. For example, he frequently moves from discussion of a three-dimensional 
(3D) "percept" to the problem of machine recognition of shapes, as if these were the same (e.g., Pizlo 2008, 180). But this seems to be carelessness. Pizlo's real conception of shape constancy is the recovery of a viewpointless 3D shape. That is, he equates shape constancy not to the perception of a shape at a slant from a viewpoint (as in Marr's 2.5-dimensional sketch; Marr 1982), but rather to the perception of shape in a viewpointless "object-centered" manner (Marr's 3D model). That is, according to Pizlo, shape constancy is a matter of recovering the unique shape of an object via an inverse inference based on its two-dimensional (2D) retinal projection. It does not concern the phenomenal experience of the surface structure of the object but is independent of viewpont: "Shape has nothing to do with viewpoint of an observer because the shape of an object is intrinsic to the object. It follows that the only way to be sure that one is studying shape, rather than depth or the orientation of surfaces, is to study shape constancy. Depth and the orientation of surfaces depend on viewpoint. Shape does not" (Pizlo 2008, 166). Pizlo's approach misconstrues the aim of shape-constancy experiments and the character of shape-constancy theorizing, by assuming that the only task involved is the one that corresponds to objective instructions: to assess the actual shape present, using background assumptions about the structure of objects in order to achieve an accurate object representation.

Leaving differences in goal and terminology aside, we can better understand Pizlo's perspective by considering further aspects of his proposals for how shape perception works. He sees shape perception as a type of inverse inference, from a 2D retinal image to a 3D shape. But he models this inference as one from a posited 3D structure to its retinal projection, taking orientation and distance into account. Thus, one way to solve shape constancy would be to consider all possible 3D objects at all possible slants and distances and choose the one that fits the retinal image (of course, for a single retinal image the solution will not be unique). Pizlo recognizes that this is not feasible, so he suggests constraints on the $3 \mathrm{D}$ objects, such as symmetry and regularity, and seeks other simplifying biases (Pizlo et al. 2010). Pizlo's notion that shape perception should be viewpointless helps explain his lack of interest in phenomenal shape. One uses the 3D model of a shape to derive its retinal projection, given its orientation and distance from the eye. In fact, Pizlo holds that distance is irrelevant to shape constancy, because it affects the size of the image but not the shape of the object $(2008,188)$. With enough constraints, such as that the stimuli are known in advance to be rectangles, shape identification is possible.

Pizlo's approach befits machine vision problems that aim to retrieve objective shape. By contrast, the experimental tradition in psychology has been interested in how shape experience arises, including various ways that phenomenal experience falls short of full constancy, and it has sought to discern the characteristics of the underlying processes from the ways in 
which constancy can be compromised. For the experimental tradition, the greater interest has been in the question of phenomenal shape constancy and its lack, with constancy of objective judgments as a secondary factor. An investigator with these interests will advisedly avoid using stimuli that allow a direct move from knowledge of the type of objective shape to the current instance of that type.

4. Size Constancy and the Geometry of Visual Space. Turning to size constancy, phenomenal factors are again salient in the experimental tradition. Many experiments rely on instructional variations between "apparent" and "objective" instructions. Some recent experiments, as well as individual attention to one's own experience, make a case for the underconstancy of phenomenal size at a distance: things, to some extent, look smaller in the distance.

The basic geometry of size perception has been known from the time of Ibn al-Haytham (eleventh century) and Descartes: for adult perceivers, perceived size depends on both visual angle and perceived distance (see Hatfield and Epstein 1979). Visual angle varies inversely with distance, but size perception does not vary with such angles alone; it relies on perceived distance. Consider an example in which a smaller object at a closer distance takes up the same visual angle as a larger object at a farther distance (fig. $3 a$ ). Assuming that visual angle is accurately recorded by the visual system (see Hershenson 1999, chap. 2) and that distance is accurately perceived, both objects are perceived with their true sizes, as with size constancy. If two objects of different size are at the same distance from the eye, then the smaller one subtends a smaller visual angle (fig. $3 b$ ); if the distance is accurately perceived, then the sizes of the two objects are accurately perceived.

After Kepler's discovery of the retinal image in the early seventeenth century, visual angle could be equated with the size of the image that an object projects onto the retina. This relation includes the previous one: larger and smaller objects at the same distance project larger and smaller retinal images, and the same object at a greater distance produces a smaller retinal projection. In the case of shape, a circle viewed straight on projects a circle, but when viewed from increasing angles it projects a family of ellipses of increasing eccentricity. These retinal facts thus far only specify projective sizes and shapes. Except when the retinal values are combined with a perception of distance and orientation, these facts do not specify a visual experience of an object with a size and shape at a distance.

In fact, theorists have disagreed over whether perceivers experience projective (retinal) values. Some spoke of sensations or appearances that correspond to the retinal projections: a given object is smaller in sensation at greater distance, and its shape in sensation varies with viewing angle. Where 
(a)

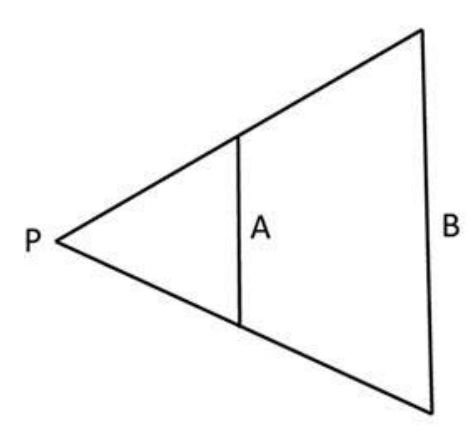

(b)
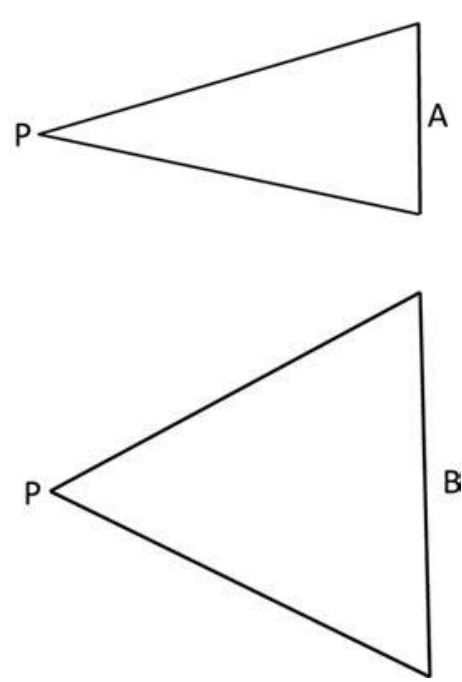

Figure 3. Diagram (a) illustrates that objects of different sizes can be placed at distances so that they project an equal visual angle. If distance is correctly perceived, then the size-distance invariance hypothesis predicts that size is correctly perceived. If object B were perceived to be at the distance of object A, then the hypothesized relation predicts that it would appear with size A. Diagram $(b)$ illustrates that objects of different sizes at the same distance project different visual angles, the larger object projecting the larger angle.

such sensations appear to be was problematic. Berkeley $(1709$, sec. 41$)$ put them "in the mind," whereas Reid $(1785,416)$ had them at "no distance from the eye."

Later, James Sully, in a widely used textbook, contended that a 2D sensation experienced in adult life is a fiction. Adults always localize and experience sensations as being at some distance (Sully 1892, 2:208, 245). This distance may be perceived more or less accurately. Concurrently, W. H. R. Rivers described how perceived size (or magnitude) is determined by retinal image size together with perceived distance:

The two chief factors on which depends the perception of the size of an object are the size of the retinal image and the estimated distance of the object. The importance of the latter factor is shown by an after-image experiment. If the after-image of an object is projected on a screen, it will be seen to change in size, becoming smaller as the screen approaches the eyes, larger as it recedes. The retinal image upon which the after-image depends 
remains constant in size, and the changes in apparent magnitude depend on the projection. Emmert measured the after-image at different distances, and found the linear size of the image equal to the linear size of the object, multiplied by the distance. (Rivers 1900, 1139-40)

In speaking of "projection," Rivers means that a subjective phenomenon, such as an afterimage, is perceived as localized at a specific location - in this case, on the surface of a screen. In Emmert's experiment, the size of the afterimage varies directly with the distance to the screen. This relation, specifying that perceived size is a function of visual angle and perceived distance, is known as "Emmert's law" (Boring 1942, 292-93, 310). Rivers generalizes the function to normal perception. The function later became known as the size-distance invariance hypothesis (see Wagner 2006, 226). Accordingly, if distance is veridically perceived, size is veridical (size constancy is attained); if distance is underperceived, size is underperceived, etc. (see fig. $3 a$ ).

Sully and Emmert invoked perceived or apparent size, but it is not clear that their empirical studies controlled for the distinction between objective and apparent judgments. From the 1950s onward, it has been standard in studies of size perception to control for observer attitude through instructional differentiation between "apparent" and "objective" judgments of size.

Granrud $(2004,2012)$ used instructional variations to investigate the role of perceptual knowledge in developmental changes for size perception at far distances. Previous literature recorded a developmental trend, with constancy improving through age 10. Two interpretations had been offered. First, according to the "perceptual learning" hypothesis, as children become better at distance perception, their phenomenal experience of the sizes of things at a distance changes (in accordance with the size-distance invariance hypothesis). Prior to learning, children experience objects at a distance with underconstancy, as smaller than they are. After learning and into adulthood, they achieve something close to full phenomenal size constancy, at least for moderate distances. Second, according to the "cognitive supplementation" hypothesis, children and adults both experience the sizes of objects at a distance with underconstancy. With development, children learn to take the distance into account cognitively, correcting their overt size responses under objective instructions to yield constancy or overconstancy, even as their experiences continue to exhibit underconstancy.

Granrud reasoned that for instructional variations to have their effect, perceivers must understand what they are asked to report. If things do look smaller in the distance, subjects should show underconstancy under objective instructions until they learn to distinguish apparent from objective. Accordingly, developmental trends toward improved constancy at far distances reflect a new ability to compensate cognitively for distance under objective 
instructions, rather than a change in phenomenal experience. To test this hypothesis, Granrud measured 5- to 10-year-old children's knowledge of the effects of distance on the appearance of size. He found that younger children did not consider that things look smaller in the distance, but that they acquired such knowledge between the ages of 5 and 10. He devised a test for distinguishing low- and high-knowledge children and then compared their responses in perceiving size at near $(6.1 \mathrm{~m})$ and far $(61 \mathrm{~m})$ distances under apparent and objective instructions.

Granrud found that both high- and low-knowledge groups showed the same degree of underconstancy for the closer objects. Farther away, under objective instructions, the high-knowledge group exhibited perfect constancy (on average), with a significant number showing overconstancy; under apparent instructions, they showed more underconstancy for far than near objects. The low-knowledge group showed considerable underconstancy for the far distance, much more so than the near; instructional variation had no effect.

Granrud found that knowledge differences alone could account for these responses. Hence, he rejected the "perceptual experience" account of developmental changes. He concluded that "adults and children have the same perceptual experience" $(2004,78)$, of apparent size diminishing with distance, and that "far-distance size constancy results from a cognitive judgment and is not a feature of perception" (89) - that is, it is not a feature of the phenomenal spatial structure of visual experience. He attributed underconstancy to a denigration of distance information for objects farther away (78).

Although Granrud ascribes phenomenal underconstancy to an error in distance perception, I have a different interpretation: that the geometry of phenomenal visual space is compressed with respect to physical space. I believe that this compression is a brute subjective fact of human vision that cannot be explained by the geometry of stimulation alone. If the sizedistance invariance hypothesis generally holds for human perception, then the fact that objects at a farther distance appear smaller does not follow from the fact that they project a smaller visual angle. A value for distance must be added. If it is systematically undervalued, we could explain Granrud's results and also account for many everyday observations. There is no mathematical reason why distance should be undervalued; it is an empirical matter whether it is.

If a human observer looks down some railroad tracks while stationed between them, the tracks converge phenomenally in a regular way. Although the precise geometry of this convergence remains under investigation, phenomenally the convergence seems regular. As they converge, the tracks remain close to phenomenally straight lines. All the same, the convergence does not fool us. We immediately recognize that the tracks do not become narrower.

If we assume that this contraction of visual space results from a systematic undervaluing of distance, we need not see this as an error. It may be 
a good thing that objects nearer at hand have greater phenomenal size than objects at a significant distance. In any case, if we accept the description of the phenomenal and cognitive facts in the previous paragraph, the experienced convergence offers an everyday example of our ability to differentiate phenomenal facts from objective spatial judgments: the tracks converge phenomenally, but we readily describe them as physically parallel (see also Hatfield 2012).

5. On Accessing Phenomenal Experience. In experimental psychology, investigators seek experimental conditions in which subjects' responses indicate the structure of their phenomenal experience. Investigators have pursued this aim for several dimensions of visual experience, including shape and size. Such efforts require experimental methods for teasing perceptual experience apart from a subject's overt or tacit beliefs about experimental conditions. Accordingly, experimenters have used specially constructed stimuli and have manipulated subjects' attitudes through instructions.

The primary evidence that these measures allow investigators to access phenomenal experience lies in the observed patterns of response. I have argued that investigations into shape and size constancy successfully access phenomenal shape and size. This conclusion depends on an interpretation of results in light of the experimental conditions. For shape constancy, under limited viewing time perceived shape varies in the expected direction. For size constancy, Granrud accounted for developmental data by distinguishing judgments of apparent and objective size. These results support experimenters' claims to distinguish phenomenal aspects of perceptual experience from beliefs about objective spatial structures.

\section{REFERENCES}

Berkeley, George. 1709. An Essay towards a New Theory of Vision. Dublin: Rhames \& Papyat. Boring, Edwin G. 1942. Sensation and Perception in the History of Experimental Psychology. New York: Appleton-Century-Crofts.

Epstein, William, Helen Bontrager, and John Park. 1962. "The Induction of Nonveridical Slant and the Perception of Shape.” Journal of Experimental Psychology 63:472-79.

Epstein, William, Gary Hatfield, and Gerard Muise. 1977. "Perceived Shape at a Slant as a Function of Processing Time and Processing Load." Journal of Experimental Psychology: Human Perception and Performance 3:473-83.

Granrud, Carl E. 2004. "Visual Metacognition and the Development of Size Constancy." In Thinking and Seeing: Visual Metacognition in Adults and Children, ed. Daniel T. Levin, 75-95. Cambridge, MA: MIT Press.

- 2012. "Judging the Size of a Distant Object: Strategy Use by Children and Adults." In Visual Experience: Sensation, Cognition, and Constancy, ed. Gary Hatfield and Sarah Allred, 13-34. Oxford: Oxford University Press.

Hatfield, Gary. 2012. "Phenomenal and Cognitive Factors in Spatial Perception." In Visual Experience: Sensation, Cognition, and Constancy, ed. Gary Hatfield and Sarah Allred, 35-62. Oxford: Oxford University Press. 
Hatfield, Gary, and William Epstein. 1979. "The Sensory Core and the Medieval Foundations of Early Modern Perceptual Theory." Isis 70:363-84.

Hershenson, Maurice. 1999. Visual Space Perception: A Primer. Cambridge, MA: MIT Press.

Leibowitz, Herschel, and L. E. Bourne. 1956. "Time and Intensity as Determiners of Perceived Shape." Journal of Experimental Psychology 51:277.

Marr, David. 1982. Vision: Computational Investigation into the Human Representation and Processing of Visual Information. San Francisco: Freeman.

Pizlo, Zygmunt. 2008. 3D Shape: Its Unique Place in Visual Perception. Cambridge, MA: MIT Press.

Pizlo, Zygmunt, Tadamasa Sawada, Yunfeng Li, Walter G. Kropatsch, and Robert M. Steinman. 2010. "New Approach to the Perception of 3D Shape Based on Veridicality, Complexity, Symmetry and Volume." Vision Research 50:1-11.

Reid, Thomas. 1785. An Inquiry into the Human Mind. 4th ed. Edinburgh: Bell \& Creech.

Rivers, W. H. R. 1900. "Vision.” In Text-Book of Physiology, vol. 2, ed. E. A. Schäfer, 1026-1148. Edinburgh: Pentland.

Rock, Irvin. 1975. Introduction to Perception. New York: Macmillan.

Stavrianos, Bertha K. 1945. "The Relation of Shape Perception to Explicit Judgments of Inclination." Archives of Psychology 296:1-94.

Sully, James. 1892. The Human Mind: A Text-Book of Psychology. 2 vols. New York: Appleton.

Thouless, Robert H. 1931. "Phenomenal Regression to the 'Real' Object." Pt. 1. British Journal of Psychology 21:339-59.

. 1932. "Phenomenal Regression to the 'Real' Object." Pt. 2. British Journal of Psychology 22:1-30.

Wagner, Mark. 2006. The Geometries of Visual Space. Mahwah, NJ: Erlbaum. 